\title{
Blood Volume Status in Patients with Chronic Fatigue Syndrome: Relation to Complaints
}

\author{
C. (Linda) M. C. van Campen, Frans C. Visser \\ Stichting CardioZorg, Hoofddorp, The Netherlands \\ Email: info@stichtingcardiozorg.nl
}

How to cite this paper: van Campen, C. (Linda) M.C. and Visser, F.C. (2018) Blood Volume Status in Patients with Chronic Fatigue Syndrome: Relation to Complaints. International Journal of Clinical Medicine, 9, 809-819.

https://doi.org/10.4236/ijcm.2018.911067

Received: November 1, 2018

Accepted: November 24, 2018

Published: November 27, 2018

Copyright $\odot 2018$ by authors and Scientific Research Publishing Inc. This work is licensed under the Creative Commons Attribution International License (CC BY 4.0).

http://creativecommons.org/licenses/by/4.0/

\begin{abstract}
Four studies have compared a possible decrease in circulating blood volume in Myalgic encephalomyelitis/chronic fatigue syndrome (ME/CFS) patients when compared to a healthy population. A more recent study has proven a correlation between $\mathrm{RBC}$ volume and $\mathrm{OI}$ in chronic OI patients without being diagnosed ME/CFS. The aim of the present study was to relate measured blood, RBC and plasma volumes (absolute and percent normalized) with the orthostatic intolerance complaints in ME/CFS patients. In the included 11 female ME/CFS patients, percentage decrease in normalized blood, RBC and plasma volume was similar for all three components: $83 \% \pm 12 \%, 83 \% \pm 12 \%$ and $83 \% \pm 11 \%$, respectively. In patients with a clinical suspicion of OI ( $\mathrm{n}=$ 7) all 3 volume components were significantly lower compared to patients without clinical suspicion of OI $(n=4)$. The difference percentage to normalized Blood volume was 77(7) vs 94(10) (p-value $<0.02$ ), difference percentage to normalized RBC volume was 76(7) vs 96(10) (p-value $<0.01$ ) and difference percentage to normalized plasma volume was $77(7)$ vs $93(10)$ (p-value $<0.05)$ in OI present versus absent. Plasma volumes were plotted against $\mathrm{RBC}$ volumes: the relation found was $\mathrm{RBC}$ volume $=0.99^{*}$ Plasma volume $+1.55 ; \mathrm{p}<0.001 ; \mathrm{r}=0.90$. In line with literature data, this pilot study shows that total blood volume and its components: RBC and plasma volume may be reduced in ME/CFS patients, especially in the presence of a clinical suspicion of OI.
\end{abstract}

\section{Keywords}

Blood Volume, Chronic Fatigue Syndrome, Orthostatic Intolerance, Red Blood Cell Volume

\section{Background}

Myalgic encephalomyelitis/chronic fatigue syndrome (ME/CFS) is amongst others 
characterized by a persistent severe fatigue, diminished exercise tolerance, post exertional malaise, muscle pain, sleep disorders and memory and concentration impairment. Furthermore, a prominent feature is that patients with ME/CFS often complain about dizziness and/or light headedness both while exercising and at rest. More than in the general population, patients experience syncope and near syncopal attacks [1] [2]. Two older studies and one more recent study have shown that the circulating blood volume in CFS patients is decreased in comparison to a healthy population [3] [4] [5]. Importantly, Farquhar et al. demonstrated [4] that there is a relation between exercise capacity and the reduction in blood volume. Another study found an inverse relation [6] between the severity of CFS symptoms and reduced red blood cell (RBC) and plasma volumes. A more recent Chinese study [7] presented evidence for a relation between $\mathrm{RBC}$ volume deficiency and the presence of orthostatic intolerance in chronic orthostatic intolerance patients and especially in postural orthostatic tachycardia syndrome (POTS) patients. Given this observation in a more general population, the aim of the present study was to relate measured blood, RBC and plasma volumes as well as the difference in percentage between volumes measured and volumes normalized with the orthostatic intolerance complaints in ME/CFS patients.

\section{Patients, Material and Methods}

Eleven female ME/CFS patients were studied from early 2010 till early 2011. ME/CFS was considered present if participants met both the 1994 International Chronic Fatigue Syndrome Study Group criteria for CFS [8] as well as the 2011 international consensus definition of ME [9]. The clinical suspicion of orthostatic intolerance (OI) was made based upon individual's feelings in the following circumstances: while waiting in line, at receptions, in choir, while shopping or at the mall, while sitting still for long periods, and when exposed to warm/stressful circumstances (e.g., summer weather; after hot showers, baths, and saunas; after episodes of fear, pain, or exposure to blood [10] [11] [12] [13]. A clinical suspicion of OI was not made if only a few of those symptoms occurred rarely or with a clear foundation. Information on the percentage of maximal predicted VO2 peak on a cardiopulmonary exercise test was available for all patients and was used to study the relation between blood volume and percentage predicted maximal VO2 peak reported in a previous study [4]

Patients underwent the standard dual isotope erythrocyte labelling technique to determine blood volume, erythrocyte and plasma volume as previously described [14]. This was done at the department of Nuclear Medicine of the Free University Hospital Amsterdam. Volumes were expressed as percentage of the mean normal reference values as described before [15]. In the remainder of this article these values will be referred to as percentage normalized volume, for blood, red blood cell and for plasma. Volumes were also expressed as absolute values (total and per kg body weight). This was done in order to be able to make 
a clear comparison to previous literature and findings. The use of clinical data for descriptive studies was approved by the ethics committee of the Slotervaart Hospital.

\section{Statistical Analysis}

Scores were tested for normal distribution using the Shapiro Wilk test in SPSS (IBM SPSS version 21). All data were normally distributed, except for the ME/CFS disease duration. Normally distributed data were presented as mean \pm $\mathrm{SD}$, the disease duration was presented as a median (IQR). Data were compared with the student's t-test for unpaired data where appropriate. A p-value of $<0.05$ was considered significantly different. Linear regression was performed on several topics like disease duration, volume measurements, age and peak VO2 results using the Graphpad Prism software (Graphpad Prism version 6.00, Graphpad software, La Jola California USA). Graphs were constructed using Graphpad Prism.

\section{Results}

11 female ME/CFS patients participated in the study. The mean age was $33 \pm 11$ years, the median duration of disease was 7 (6 - 10) years. Percentage of normalized blood volume, RBC volume and plasma volume was similar in the group as a whole for all three components: $83 \% \pm 12 \%, 83 \% \pm 12 \%$, and $83 \% \pm 11 \%$, respectively. Total blood, RBC and plasma volumes were $3707 \pm 818 \mathrm{ml}, 1280 \pm$ $316 \mathrm{ml}$ and $2427 \pm 509 \mathrm{ml}$, respectively. Blood, RBC and plasma volumes expressed as $\mathrm{ml}$ per $\mathrm{kg}$ were $59 \pm 8 \mathrm{ml} / \mathrm{kg}, 20 \pm 3 \mathrm{ml} / \mathrm{kg}$ and $39 \pm 5 \mathrm{ml} / \mathrm{kg}$, respectively. Figure 1 shows the difference between measured and normalized volumes

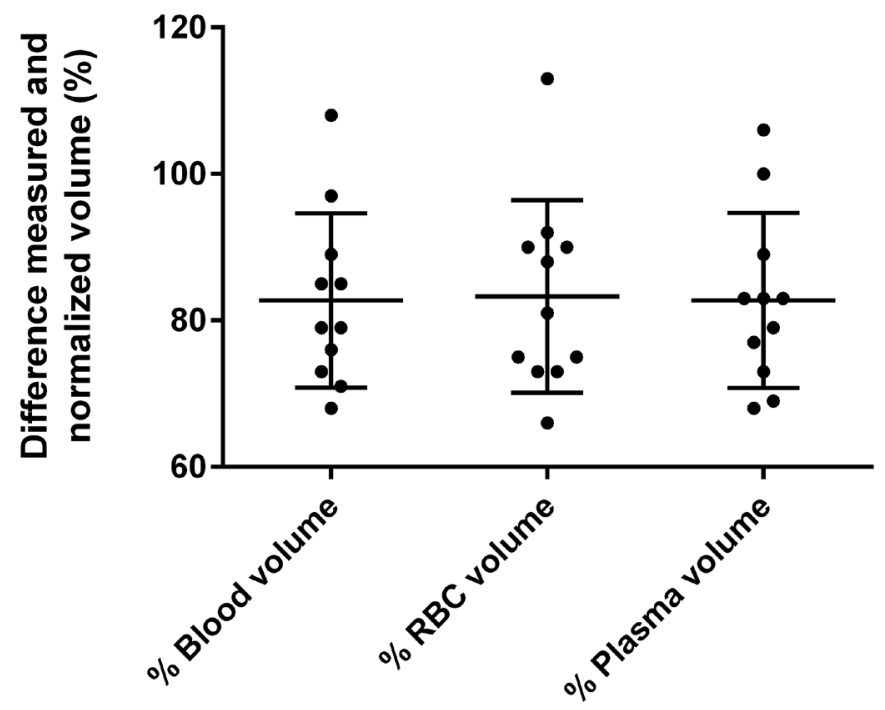

Figure 1. Difference between measured and normalized blood volume components expressed as a percentage of the normalized blood volume components for the whole group. $\%$ = percentage; $\mathrm{RBC}=$ red blood cell. 
expressed as a percentage of the normalized volume for blood, RBC and plasma for the whole group.

Of the 11 patients, 4 had no clinical suspicion of OI complaints whereas 7 did have a clinical suspicion of OI complaints present. All 7 patients with a clinical suspicion of OI were homebound and all 4 patients without a clinical suspicion of OI were not. Of the 7 patients with a clinical suspicion of OI, only 2 had POTS (postural orthostatic tachycardia syndrome), no patient had orthostatic hypotension (OI), vasovagal syncope or neurogenic mediated syncope.

Table 1 shows the baseline data and blood volume component data in patients without $(\mathrm{n}=4)$ and with $(\mathrm{n}=7)$ a clinical suspicion of OI. No differences were found between the two groups with respect to length, weight and disease duration. Patients with OI were also significantly younger $(\mathrm{p}<0.05)$. When volumes were measured and expressed per $\mathrm{kg}$, a significant difference was seen in blood volume ( $66 \pm 9$ vs $55 \pm 4: \mathrm{p}<0.05)$ and in $\mathrm{RBC}$ volume $(23 \pm 3$ vs $19 \pm 1: \mathrm{p}<$ 0.05). A trend was shown in the plasma volume per $\mathrm{kg}$ (43 \pm 6 vs $37 \pm 9: \mathrm{p}=$ 0.09). Patients with a clinical suspicion of OI were found to have lower values whereas patient without a clinical suspicion of OI were found to have higher values. When these differences between measured and normalized volumes were expressed as percentages of that normalized volume, a significant difference was found when subjects both with and without OI were compared across all 3 normalized volume components: blood volume $(94 \% \pm 10 \%$ vs $77 \% \pm 7 \%$ : $\mathrm{p}<0.02)$, RBC volume $(96 \% \pm 10 \%$ vs $76 \% \pm 7 \%: \mathrm{p}<0.01)$ and plasma volume $(93 \% \pm$ $10 \%$ vs $77 \% \pm 7 \%: \mathrm{p}<0.05)$. The measured volume components in $\mathrm{ml} / \mathrm{kg}$ are graphically visualized in Figure 2(a) whereas differences between measured and normalized volume components expressed as a percentage of normalized volumes is visualized in Figure 2(b).

No significant correlation was found between the disease duration and the percentage of normalized volume components or the absolute measured volume

Table 1. Baseline and volume components.

\begin{tabular}{cccc}
\hline & OI absent & OI present & p-value \\
Number & 4 & 7 & $<0.05$ \\
Age (years) & $44 \pm 3$ & $27 \pm 9$ & ns \\
Length (cm) & $173 \pm 8$ & $170 \pm 10$ & ns \\
Weight (kg) & $65 \pm 8$ & $61 \pm 11$ & ns \\
Disease duration (years) & $9(8-11)$ & $7(5.5-8.5)$ & $<0.05$ \\
Blood volume per kg (ml) & $66 \pm 9$ & $55 \pm 4$ & $<0.05$ \\
RBC volume per kg (ml) & $23 \pm 3$ & $19 \pm 1$ & 0.09 \\
Plasma volume per kg (ml) & $43 \pm 6$ & $37 \pm 3$ & $<0.02$ \\
Percentage norm blood volume (\%) & $94 \pm 10$ & $77 \pm 7$ & $<0.01$ \\
Percentage norm RBC volume (\%) & $96 \pm 10$ & $76 \pm 7$ & $<0.05$ \\
Percentage norm RBC volume (\%) & $93 \pm 10$ & $77 \pm 7$ &
\end{tabular}

Norm = normalized; $\mathrm{OI}=$ orthostatic intolerance; $\mathrm{RBC}=$ red blood cell; yrs = years. 


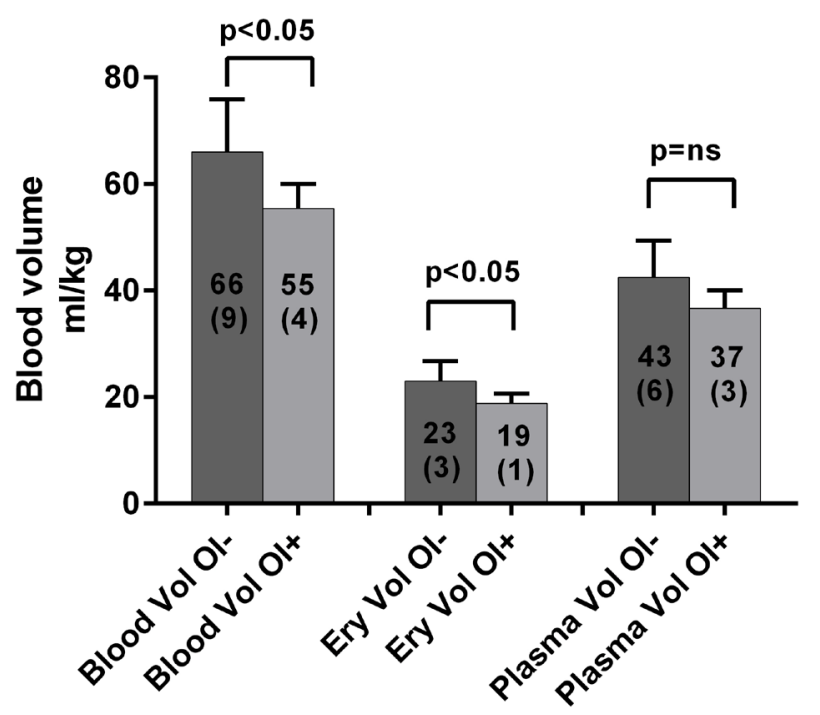

(a)

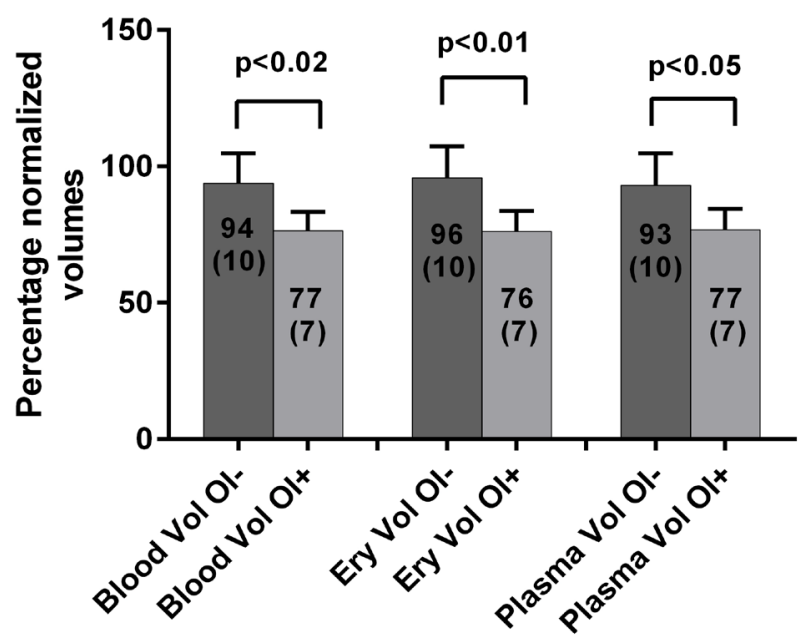

(b)

Figure 2. (a) Blood, RBC and plasma volume per kg body weight and the absence or presence of orthostatic intolerance. OI = orthostatic intolerance; - = absent; + = present; $\mathrm{RBC}=$ red blood cell; Vol = volume. (b) Blood, RBC and plasma volume difference measured and normalized expressed as a percentage of the normalized volume and the absence or presence of orthostatic intolerance. OI $=$ orthostatic intolerance; - = absent; + = present; $\mathrm{RBC}=$ red blood cell; $\mathrm{Vol}=$ volume.

components (neither for blood volume, RBC volume or plasma volume), as is shown in Table 2. Plasma volumes were plotted against RBC volumes. The following mathematical relation was given by the following formula: $\mathrm{RBC}$ volume $=$ $0.99^{\star}$ Plasma volume $+1.55 ; \mathrm{p}<0.001 ; \mathrm{r}=0.90$. This is shown in Figure 3.

No significant correlation was found between blood volume in $\mathrm{ml} / \mathrm{kg}$ and the percentage of VO2 peak (Figure 4(a)). However, when the measured blood volume was expressed as a percentage of a calculated normalized blood volume, 
Table 2. Correlation disease duration and volume components absolute per kg and percentage difference between measured and normalized volumes.

\begin{tabular}{cccc}
\hline & Regression line & $\mathrm{r}$ & p-value \\
\hline Disease duration vs Norm BV\% & $\mathrm{Y}=1.023 \mathrm{X}+74.45$ & 0.27 & $\mathrm{~ns}$ \\
Disease duration vs Norm RBC V\% & $\mathrm{Y}=1.107 \mathrm{X}+74.31$ & 0.27 & $\mathrm{~ns}$ \\
Disease duration vs Norm PV\% & $\mathrm{Y}=0.8748 \mathrm{X}+75.65$ & 0.23 & $\mathrm{~ns}$ \\
Disease duration vs BV/kg & $\mathrm{Y}=-0.2604 \mathrm{X}+61.38$ & 0.10 & $\mathrm{~ns}$ \\
Disease duration vs RBC V/kg & $\mathrm{Y}=-0.0730 \mathrm{X}+20.95$ & 0.07 & $\mathrm{~ns}$ \\
Disease duration vs PV/kg & $\mathrm{Y}=-0.2955 \mathrm{X}+41.21$ & 0.17 & $\mathrm{~ns}$ \\
\hline
\end{tabular}

$\mathrm{BV}=$ Blood Volume; $\mathrm{RBC}$ V = red blood cell volume; norm = normalized; PV = Plasma Volume; \% = percentage; vs $=$ versus.

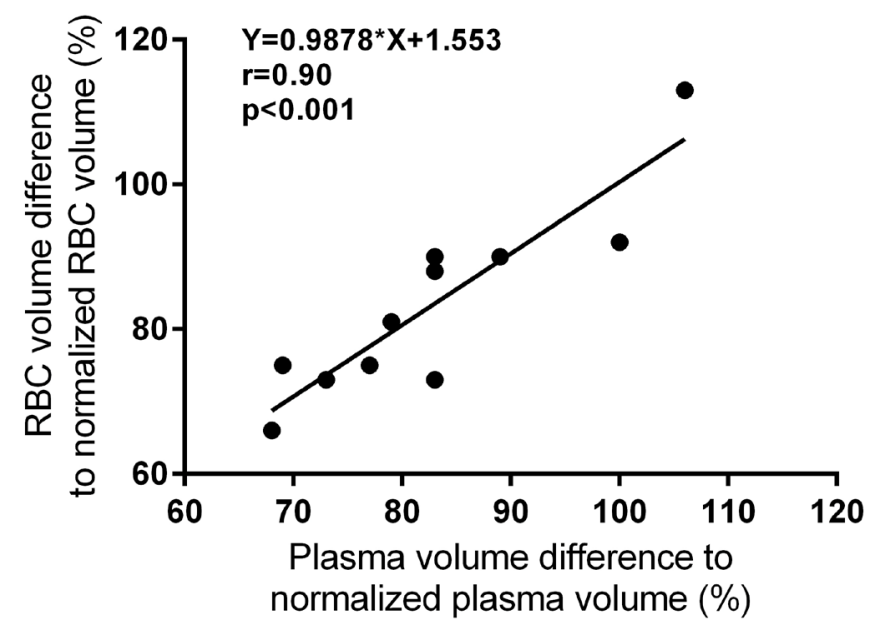

Figure 3. Relation RBC volume and plasma volume: difference between measured volume and normalized volume expressed as a percentage of the normalized volume. $\%=$ percentage; $\mathrm{RBC}=$ red blood cell.

a significant relation was found (Figure 4(b)). As the patients with a clinical suspicion of OI were homebound, compared to those without a clinical suspicion of OI, this difference in severity was confirmed by a significant difference in percentage of predicted VO2 peak $(95 \% \pm 10 \%$ in the group without OI vs $67 \% \pm$ $17 \%$ in the group with OI, $\mathrm{p}<0.02)$.

\section{Discussion}

The characterizing elements of patients suffering from the chronic fatigue syndrome (CFS) are fatigue, orthostatic intolerance, and numerous somatic complaints. The shortcoming of a detectable routine laboratory test in order to obtain the diagnoses of CFS/ME has led to assumptions that CFS may be either a trivial illness or a psychosomatic disorder. Blood volume measurements have traditionally been used in the management of polycythemia but not in the evaluation of CFS until the early 90s [16]. Limited studies on blood volume have been performed in ME/CFS patients. 


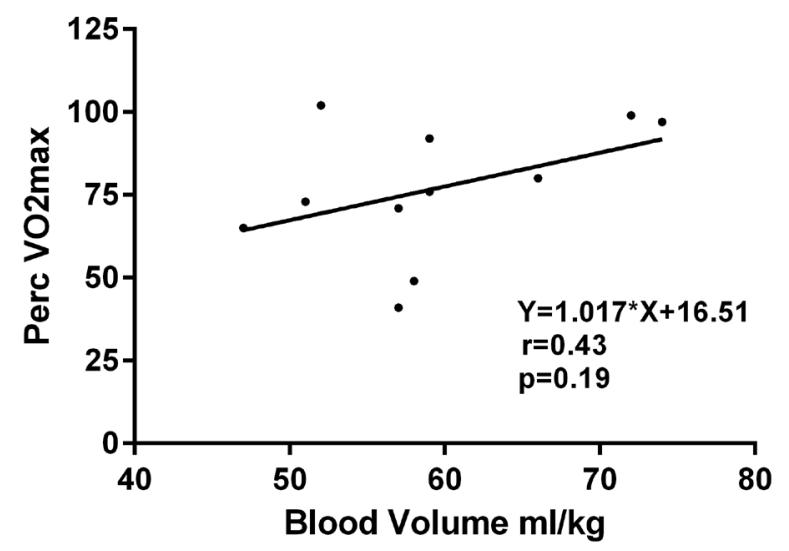

(a)

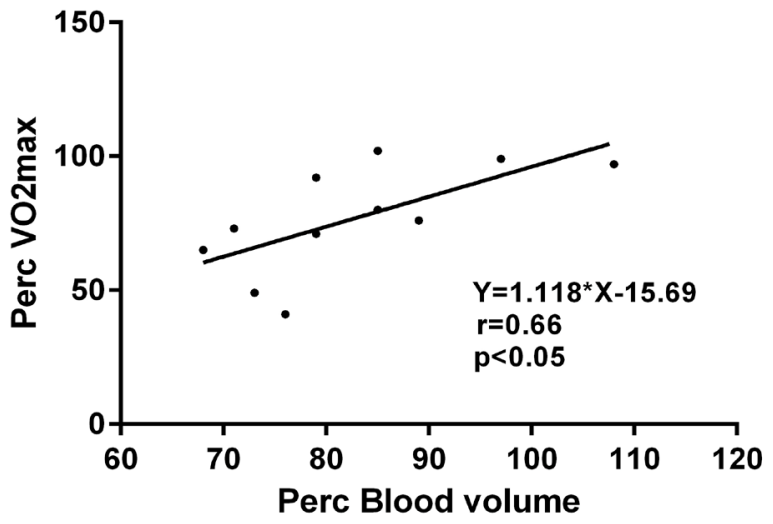

Figure 4. (a) Relation between measured blood volume in $\mathrm{ml} / \mathrm{kg}$ versus percentage of predicted VO2 peak. Perc = percentage; $\mathrm{VO} 2$ = maximal oxygen consumption. (b) Relation between the measured blood volume expressed as a percentage of the normalized calculated blood volume versus the predicted VO2 peak. Perc = percentage; VO2 = maximal oxygen consumption.

The main finding of this study was that a significantly lower measured blood volume per $\mathrm{kg}$ and $\mathrm{RBC}$ volume per $\mathrm{kg}$ as well as normalized percentage of blood, RBC and plasma volumes were found in patients with a clinical suspicion of OI as compared to patients without a clinical suspicion of OI. The data of RBC volumes are similar to a study in which only OI patients were investigated [7]. Lin and colleagues found a RBC volume between 78\% (POTS patients) and $85 \%$ (non-POTS patients), compared to a reference RBC volume of $100 \%$. A decrease of RBC volume in OI is in line with our study where patients with OI had a 77\% normalized RBC volume.

Another finding of our study was the significant relation between the measured blood volume expressed as percentage of a normalized blood volume and the percentage of predicted VO2 peak. This confirmed the finding of Farquhar and colleagues [4] They found a trend to a lower blood volume of 17 CFS patients versus 17 controls, which reached no significance $(p=0.08)$. The blood volumes found in control subjects of $64.2 \pm 2.5 \mathrm{ml} / \mathrm{kg}$ were in line with the blood 
volumes result in the group of ME/CFS patients without OI of $66 \pm 9 \mathrm{ml} / \mathrm{kg}$ and the blood volume in CFS patients found in that trial of $58.3 \pm 2.1 \mathrm{ml} / \mathrm{kg}$ was similar to the finding in our study in ME/CFS patients with OI of $55 \pm 4 \mathrm{ml} / \mathrm{kg}$. This was in line with findings of two earlier published studies which described this finding in healthy female subjects [17] and in healthy male subjects [18]. Both those trials in healthy subjects found a decline of total blood volume with increasing age. This fact has been described as early as 1937 by Gibson and colleagues [19]. The fact that we found a significantly lower blood volume in the patients with orthostatic intolerance symptoms, who were younger than the patient group without a clinical suspicion of OI, might suggest that the absolute difference in blood volume might have been bigger, if patients had been age matched.

Streeten and colleagues [3] found that RBC volumes were lower in 12 female CFS patients as compared to RBC volumes in female control subjects. In contrast plasma and total blood volume were not significantly different from control subjects. Their indexed blood volume of 2221 (SE 65) was similar to ours 2143 (SE 98), as was the RBC volume 774 (SE 26) and 1439 (SE 59), and the plasma volume of 738 (SE 39) and 1405 (SE 62) in the study of Streeten et al. in comparison to our study. Information on clinical symptoms of orthostatic intolerance were not clearly stated, however information on blood pressure during prolonged standing (up to 84 minutes), suggested orthostatic hypotension (systolic blood pressure decline $\geq 20 \mathrm{mmHg}$ ) without complaints during standing or in history of 7 of 12 patients.

Hurwitz and colleagues [5] found that in 30 more severe CFS and in 26 non-severe CFS patients total blood volume (TBV), RBC and plasma volume were not significantly different from 21 sedentary controls (TBV: $57.3 \pm 1.7$ vs $61 \pm 1.8$ vs $63.1 \pm 2.0$; PV $37.2 \pm 1.1$ vs $38.8 \pm 1.1$ vs $41 \pm 1.3$; RBCV $20.2 \pm 0.8$ vs $22.2 \pm 0.8$ vs $22.1 \pm 0.9 \mathrm{ml} / \mathrm{kg}$ ) This is similar to the findings in the study at hand where the mean TBV was $59 \pm 8$, the mean PV was $39 \pm 5$ and the mean RBCV was $20 \pm 3 \mathrm{ml} / \mathrm{kg}$. However, when recalculating the difference from ideal volumes, percent total blood volume, percent plasma volume and percent RBC volume were significantly different from sedentary controls and also lower in severe CFS patients than compared to less severe CFS patients (TBV\%. $-9.7 \pm 2$ vs $-2 \pm 2$ vs $8.1 \pm 2.0 ;$ PV\% $-7.6 \pm 2.4$ vs $-0.1 \pm 2.5$ vs $12.1 \pm 2.8$ and $\mathrm{RBCV} \%$ $-13.3 \pm 2.3$ vs $-5.8 \pm 2.4$ vs $1.6 \pm 2.8$ ) In our study, differences in percentage of normalized volumes were much larger in the patients with a clinical suspicion of OI. Hurwitz and et al. [5] also looked into maximal oxygen consumption at exercise but found no difference in the three groups (with a difference from predicted VO2 $\max$ of $-25.1 \pm 3.2$ vs $-32.7 \pm 2.9$ vs $-25.7 \pm 3.3$ in severe CFS, non-severe CFS and sedentary controls respectively). This is in contrast to the study at hand. Though, numbers were expressed in a similar manner (difference from predicted VO2 peak of $100 \%)-6 \% \pm 9 \%$ in the patients without a clinical suspicion of OI and $-33 \% \pm 16 \%$ in the group with a clinical suspicion of OI. They did not compare the oxygen consumption results with the volume results. 
Newton and colleagues [6] showed only a trend in lower blood volumes versus controls. However they found that $68 \%$ of a group of 41 CFS patients had a normalized RBC volume below the lower limit of normal of $<95 \%$. A normalized plasma volume below the lower limit of normal of $<95 \%$ was present in $32 \%$ of the studied 41 CFS patients. This suggests a difference in reduction between plasma and $\mathrm{RBC}$ volumes. In the present study the reduction was similar in RBC as well as plasma volumes. The slope of the linear regression between plasma and $\mathrm{RBC}$ volumes was almost identical to the line of identity. Furthermore, in the present study only one patient had a RBC volume $>95 \%$. This low number may be due to the small sample size of the study. The TBV in the study was $4236 \pm$ $139 \mathrm{ml}$ in $41 \mathrm{CFS}$ patients and $4396 \pm 180 \mathrm{ml}$ in 10 healthy controls. This was in line with the TBV value of patients without a clinical suspicion of OI in our study with $4281 \pm 821$. The TBV in the patients with a clinical suspicion of OI was significantly lower with $3380 \pm 609 \mathrm{ml}$. This study showed no correlation between disease duration and cardiac volumes existed, suggesting that deconditioning was not the cause of those abnormalities. This is in line with the finding of our study with respect to disease duration and volume measurements.

In none of the above mentioned studies, a relation of the volume components with OI symptoms was explored.

\section{Limitations}

In this study, matched control subjects were not assessed for circulating blood volume measurements, and thus the data presented here should be considered a preliminary report. Since significant reduction of total and RBC blood volume may be among the few objectively demonstrable laboratory abnormalities in the majority of ME/CFS patients with a clinical suspicion of OI or if demonstrated by a tilt table test, we believe that further studies are warranted.

\section{Conclusion}

In line with data in previous literature, this pilot shows that total blood volume and its components: RBC and plasma volume may be reduced in CFS patients. We confirmed the finding that a relation between blood volume and percentage predicted peak VO2 was present. This was supported by the finding that a lower blood volume had a worse outcome on cardiopulmonary exercise testing. One other study looked into the relation of blood volume and orthostatic intolerance, results of which are in line with ours, although the patient population studied was one of chronic orthostatic incompetence, with similar complaints as in ME/CFS, but without the diagnosis made for ME/CFS $\{$ Lin, 2014 \#752\}.

\section{Acknowledgements}

We would like to thank M Croon for careful review of the manuscript.

\section{Conflicts of Interest}

The authors declare no conflicts of interest regarding the publication of this paper. 


\section{Funding}

No financial support was obtained.

\section{Author Agreement/Declaration}

All authors have seen and approved the final version of the manuscript being submitted. They warrant that the article is the authors' original work, hasn't received prior publication and isn't under consideration for publication elsewhere.

\section{References}

[1] Ulas, U.H., et al. (2010) Comorbid Health Conditions in Women with Syncope. Clinical Autonomic Research, 20, 223-227. https://doi.org/10.1007/s10286-010-0070-x

[2] Rowe, P.C., et al. (1995) Is Neurally Mediated Hypotension an Unrecognised Cause of Chronic Fatigue? Lancet, 345, 623-624. https://doi.org/10.1016/S0140-6736(95)90525-1

[3] Streeten, D.H., Thomas, D. and Bell, D.S. (2000) The Roles of Orthostatic Hypotension, Orthostatic Tachycardia, and Subnormal Erythrocyte Volume in the Pathogenesis of the Chronic Fatigue Syndrome. The American Journal of the Medical Sciences, 320, 1-8. https://doi.org/10.1016/S0002-9629(15)40790-6

[4] Farquhar, W.B., et al. (2002) Blood Volume and Its Relation to Peak $\mathrm{O}_{2}$ Consumption and Physical Activity in Patients with Chronic Fatigue. American Journal of Physiology-Heart and Circulatory Physiology, 282, H66-H71. https://doi.org/10.1152/ajpheart.2002.282.1.H66

[5] Hurwitz, B.E., et al. (2010) Chronic Fatigue Syndrome: Illness Severity, Sedentary Lifestyle, Blood Volume and Evidence of Diminished Cardiac Function. Clinical Science (London), 118, 125-135. https://doi.org/10.1042/CS20090055

[6] Newton, J.L., et al. (2016) Reduced Cardiac Volumes in Chronic Fatigue Syndrome Associate with Plasma Volume but Not Length of Disease: A Cohort Study. Open Heart, 3, e000381. https://doi.org/10.1136/openhrt-2015-000381

[7] Lin, C.J., Chu, Y.K. and Chern, C.M. (2014) RBC Volume Deficiency in Patients with Excessive Orthostatic Decrease in Cerebral Blood Flow Velocity. Journal of the Chinese Medical Association, 77, 174-178. https://doi.org/10.1016/j.jcma.2014.01.005

[8] Fukuda, K., et al. (1994) The Chronic Fatigue Syndrome: A Comprehensive Approach to Its Definition and Study. International Chronic Fatigue Syndrome Study Group. Annals of Internal Medicine, 121, 953-959. https://doi.org/10.7326/0003-4819-121-12-199412150-00009

[9] Carruthers, B.M., et al. (2011) Myalgic Encephalomyelitis: International Consensus Criteria. Journal of Internal Medicine, 270, 327-338. https://doi.org/10.1111/j.1365-2796.2011.02428.x

[10] The National Academies Collection (2015) Beyond Myalgic Encephalomyelitis/Chronic Fatigue Syndrome: Redefining an Illness. Washington DC.

[11] Thieben, M.J., et al. (2007) Postural Orthostatic Tachycardia Syndrome: The Mayo Clinic Experience. Mayo Clinic Proceedings, 82, 308-313. https://doi.org/10.1016/S0025-6196(11)61027-6

[12] Grubb, B.P. (2005) Clinical Practice. Neurocardiogenic Syncope. New England Journal of Medicine, 352, 1004-1010. https://doi.org/10.1056/NEJMcp042601 
[13] Raj, S.R. (2013) Postural Tachycardia Syndrome (POTS). Circulation, 127, 2336-2342. https://doi.org/10.1161/CIRCULATIONAHA.112.144501

[14] Fairbanks, V.F., et al. (1996) Measurement of Blood Volume and Red Cell Mass: Re-Examination of 51Cr and 125I Methods. Blood Cells, Molecules and Diseases, 22, 169-186; discussion 186a-186g.

[15] Retzlaff, J.A., et al. (1969) Erythrocyte Volume, Plasma Volume, and Lean Body Mass in Adult Men and Women. Blood, 33, 649-661.

[16] Streeten, D.H. and Anderson Jr., G.H. (1992) Delayed Orthostatic Intolerance. Archives of Internal Medicine, 152, 1066-1072. https://doi.org/10.1001/archinte.1992.00400170138025

[17] Jones, P.P., Davy, K.P., DeSouza, C.A., van Pelt, R.E. and Seals, D.R. (1997) Absence of Age-Related Decline in Total Blood Volume in Physically Active Females. American Journal of Physiology, 272, H2534-H2540.

https://doi.org/10.1152/ajpheart.1997.272.6.H2534

[18] Davy, K.P. and Seals, D.R. (1994) Total Blood Volume in Healthy Young and Older Men. Journal of Applied Physiology, 76, 2059-2062.

https://doi.org/10.1152/jappl.1994.76.5.2059

[19] Gibson, J.G. and Evans Jr., W.A. (1937) Clinical Studies of the Blood Volume. II. The Relation of Plasma and Total Blood Volume to Venous Pressure, Blood Velocity Rate, Physical Measurements, Age and Sex in Ninety Normal Humans. Journal of Clinical Investigation, 16, 317-328. https://doi.org/10.1172/JCI100860 